\title{
Hepcidin: inflammation versus hypoxia
}

\author{
F. del Campo • C. Zamarrón
}

Received: 14 July 2014 / Revised: 17 July 2014 / Accepted: 21 August 2014 / Published online: 5 September 2014

(C) Springer-Verlag Berlin Heidelberg 2014

The finding of periodic leg movements (PLM) during polysomnographic recordings is a relatively common phenomenon. PLM is associated with a variety of sleep disorders [1, 2] and other diseases. An association between obstructive sleep apnea (OSA) and PLM [3-5] has been clearly established. However, the prevalence of PLM in OSA patients is highly variable, and the clinical relevance of this association is not yet well known. Moreover, some controversy exists regarding the relationship of PLM with OSA treatments such as CPAP or mandibular advancement prosthesis [6].

The clinical and polysomnographic profile of the association between OSA and PLM is yet to be fully determined. It frequently occurs in women and is associated with severe OSA. However, it has also been related with upper airway resistance syndrome. In addition, it has been observed in the first part of the night or during REM stage.

Although the pathophysiological mechanisms of the PLMOSA are not well known [7], it has been suggested that they probably differ from the association of PLM with other diseases [8]. For several years, attention has focused on the possibility that iron metabolism may play a role. In this regard, it has been observed that restless leg syndrome (RLS), a disease often related with PLM, is especially prevalent in situations of iron deficiency or impaired iron metabolism, such as pregnancy or advanced chronic renal failure.

F. del Campo

Hospital Rio Hortega Valladolid, University of Valladolid,

Valladolid, Spain

C. Zamarrón

Servicio de neumología, Hospital Clínico Universitario,

Santiago de Compostela, Spain

F. del Campo $(\bowtie)$

Departmento de Medicina, Dermatología y Toxicología, Hospital

Río Hortega, Universidad de Valladolid, Valladolid, Spain

e-mail: fsas@telefonica.net
Nevertheless, RLS has also been found in patients with normal iron levels.

The majority of patients with RLS have normal blood levels of ferritin, thus suggesting a causal mechanism involving the availability of iron in the central nervous system. The indications are that a dysfunction in iron transport to the central nervous system and dopamine neurotransmission abnormalities is implicated. However, few studies exist assessing this disorder in patients with OSA. O'Brien et al. [9] evaluated the association between iron levels, PLM, and sleepiness in patients with OSA but could not confirm the hypothesis that OSA patients had an iron deficit nor that a subsequent dopamine dysfunction leads to PLM and daytime sleepiness.

Iron deficiency and alterations in iron transport are related to many chronic diseases. In recent years, there has been great interest in a new hormone, hepcidin $[10,11]$, as a result of its role in iron metabolism. Hepcidin is a liver-derived peptide acting as a main regulator of body iron homeostasis by controlling iron uptake in the duodenum and its storage in macrophages. This hormone also participates in the immune and inflammatory mechanisms. Hepcidin acts by regulating iron metabolism between various organs such as the duodenum, liver, and bone marrow.

A number of studies have found a potential role of hepcidin in RLS $[12,13]$, especially in association with chronic kidney disease, pregnancy, obesity, and iron-deficiency anemia. It has been speculated that inflammation produces an iron deficiency in the central nervous system mediated by hepcidin. The regulation of hepcidin is complex and is modulated by iron levels, erythropoietic activity, hypoxia, and inflammation. Systemic hypoxia reduces hepcidin production in the liver. Although the mechanism is not yet clear, hypoxia-induced factors seem to play a role. Inflammation seems to cause an increase in hepcidin peripherally and a decrease in the central nervous system. Factors involved in inflammation such as 
interleukin-6 may be responsible for the stimulation of hepcidin synthesis.

In one of the first analyses of the possible role of hepcidin in sleep disorders, Kanbay et al. [14] proposed the hypothesis that hepcidin, through the mediation of the interleukin-6, could promote the occurrence of insulin-resistance syndrome in OSA patients. Despite this hypothesis and the increased interest in this hormone, little research has been carried out on the role of hepcidin in respiratory disease. As yet, only a small number of authors point to hepcidin as an emerging marker $[14,15]$.

In light of this, the publication by Abakay et al. [16] in this issue is of great interest, as it analyzes the relationship between hepcidin levels, iron status, and PLM index in OSA patients. This study points out three important aspects. Firstly, that OSA is associated with high levels of hepcidin compared to the control group. Secondly, that these levels are increased in relation to the OSA severity and, finally, that serum hepcidin levels were significantly correlated with PLM index. Thus, it is suggested that hypoferremia induces PLM in patients with OSA, as a result of OSA-induced inflammation.

This paper aims to contribute to a better understanding of the pathophysiology of PLM in patients with OSA. Although enough data is not yet available in the literature to suggest hepcidin as a useful inflammatory marker in the diagnosis or prognosis of the disease, this study raises interesting issues.

Given that iron is an essential element for most organisms and plays a central role in oxygen transport, hypoxia-induced factors associated with iron deficiency could produce a down regulation of hepcidin. Indeed, inflammation and hypoxia are two of the factors involved in the hepcidin regulation. With respect to respiratory disease, studies on hepcidin levels have been performed involving two low-grade inflammation diseases, COPD and OSA. However, these respiratory diseases have two different models of hypoxia (chronic versus chronic intermittent). Systemic inflammation and oxidative stress in COPD and OSA may predispose to cardiovascular disease. Moreover, hypoxia and iron deficiency have very similar consequences at the molecular level. Abakay et al. [16] attributed elevated hepcidin levels to inflammation, but they did not determine cytokine levels. In an earlier study, Duru et al. [17] found a decrease in hepcidin levels in COPD patients, which they attributed to hypoxia. In a subsequent study [18] involving also patients with COPD exacerbation and determination of IL-6 levels, these authors found that COPD exacerbation led to normalization of hepcidin levels relative to the control group, which is due to inflammation in COPD exacerbations.
Further research is necessary to adequately characterize the clinical importance of hepcidin in pathologies that share many common aspects, such as OSA and COPD.

\section{References}

1. Karatas M (2007) Restless legs syndrome and periodic limb movements during sleep: diagnosis and treatment. Neurologist 13:294-301

2. Warnes H, Dinner DS, Kotagal P, Burgess RC (1993) Periodic limb movements and sleep apnoea. J Sleep Res 2:38-44

3. Scharf SM, Tubman A, Smale P (2005) Prevalence of concomitant sleep disorders in patients with obstructive sleep apnea. Sleep Breath 9:50-56

4. Chervin RD (2001) Periodic leg movements and sleepiness in patients evaluated for sleep-disordered breathing. Am J Respir Crit Care Med 164:1454-1458

5. Al-Alawi A, Mulgrew A, Tench E, Ryan CF (2006) Prevalence, risk factors and impact on daytime sleepiness and hypertension of periodic leg movements with arousals in patients with obstructive sleep apnea. J Clin Sleep Med 2:281-287

6. Baran AS, Richert AC, Douglass AB (2003) Change in periodic limb movement index during treatment of obstructive sleep apnea with continuous positive airway pressure. Sleep 26:717-720

7. Roux FJ (2013) Restless legs syndrome: impact on sleep-related breathing disorders. Respirology 18:238-245

8. Merlino G, Gigli GL (2012) Sleep related movement disorders. Neurol Sci 33:491-513

9. O'Brien LM, Koo J, Fan L et al (2009) Iron stores, periodic leg movements, and sleepiness in obstructive sleep apnea. J Clin Sleep Med 05:525-531

10. Park CH, Valore EV, Waring AJ, Ganz T (2001) Hepcidin, a urinary antimicrobial peptide synthesized in the liver. J Biol Chem 276: 7806-7810

11. Lago F, Dieguez C, Gomez-Reino J, Gualillo O (2007) The emerging role of adipokines as mediators of inflammation and immune responses. Cytokine Growth Factor Rev 18:313-325

12. Clardy SL, Wang X, Boyer PJ, Earley CJ, Allen RP, Connor JR (2006) Is ferroportin hepcidin signaling altered in restless legs syndrome? J Neurol Sci 247:173-179

13. Weinstock LB, Walters AS, Paueksakon P (2012) Restless legs syndrome - theoretical roles of inflammatory and immune mechanisms. Sleep Med Rev 16(4):341-354

14. Kanbay A, Hasanoglu HC (2007) A new prognostic marker for obstructive sleep apnea: hepcidin. Med Hypotheses 69(6):1381-1382

15. Kapoor S (2013) Emerging new biomarkers in obstructive sleep apnea. Sleep Breath 17:453-454

16. Abakay O, Abakay A, Palanci Y, Yuksel H, Sen HS, Eviylaoglu O, Tanrikulu AC (2013) Relationship between hepcidin levels and periodic limb movement disorder in patients with obstructive sleep apnea syndrome. Sleep Breath xxxxxx

17. Duru S, Bilgin E, Ardiç S (2012) Hepcidin: a useful marker in chronic obstructive pulmonary disease. Ann Thorac Med 7:31-35

18. Duru S, Yuce G, Sarinc S, Kaplan T, Erdem M, Ucar F, Ardic S (2013) The relationship between serum hepcidin level and severity of chronic obstructive pulmonary disease. Health Med 7(5):1672-1679 